MR JAKOB KÜMMEL (Orcid ID : 0000-0002-8591-7509)

Received Date : 25-Oct-2016

Revised Date : 09-Feb-2017

Accepted Date : 27-Feb-2017

Article type : Original Article

\title{
Title: Conditioning hops increase triceps surae muscle force and Achilles tendon strain energy in the stretch-shortening cycle
}

\author{
Jakob Kümmel ${ }^{1}$, Neil J. Cronin ${ }^{2}$, Andreas Kramer ${ }^{1}$, Janne Avela ${ }^{2}, \&$ Markus Gruber $^{1}$
}

1 Sensorimotor Performance Lab, Department of Sport Science, University of Konstanz, Konstanz, Germany

${ }^{2}$ NeuroMuscular Research Center, Unit of Biology of Physical Activity, Faculty of Sport and Health Sciences, University of Jyväskylä, Jyväskylä, Finland.

Running Head: Conditioning hops increase SSC efficacy

\section{Corresponding Author:}

Jakob Kümmel

FG Sportwissenschaft

Universität Konstanz

78457 Konstanz, Germany

Email: jakob.kuemmel@uni-konstanz.de

Phone: +49 (0) 7531 88-4213

Fax: +49 (0) $753188-2372$

This article has been accepted for publication and undergone full peer review but has not been through the copyediting, typesetting, pagination and proofreading process, which may lead to differences between this version and the Version of Record. Please cite this article as doi: $10.1111 / \mathrm{sms} .12870$

This article is protected by copyright. All rights reserved. 


\begin{abstract}
Post-activation potentiation can improve athletic performance, but the underlying mechanisms are poorly understood. This study investigated the effect of conditioning hops on triceps surae muscle force and tendon strain and its contribution to potentiated stretchshortening cycle (SSC) performance. Thirty-two subjects participated in two experiments. In both experiments, subjects performed three drop jumps (DJs) after prior conditioning with 10 maximal hops, three unconditioned DJs served as control. Ground reaction forces, kinematics, and triceps surae electromyographic activity were recorded. Ultrasound imaging was used to determine fascicle lengths of the gastrocnemius and soleus muscles (experiment 1) and the length of the Achilles tendon (experiment 2) during the DJs. DJ height after the conditioning hops was significantly higher compared to control DJs (experiment $1:+12 \%$ and experiment $2:+19 \%$ ). A significantly shorter gastrocnemius fascicle length during the DJs performed after the conditioning hops coincided with an increased force acting on the triceps surae muscle. Moreover, the triceps surae muscle-tendon unit (MTU) showed increased energy absorption during the eccentric phase of the DJs, and increased energy release during the concentric phase. The second experiment revealed a higher Achilles tendon strain in DJs performed after the conditioning hops compared to control DJs. No significant differences in muscle activities were observed. The shorter fascicle length in gastrocnemius and the larger Achilles tendon strain facilitated MTU energy transfer from the eccentric to the concentric phase during the DJ. Thereby, conditioning hops improved SSC efficacy and DJ performance.
\end{abstract}

Keywords: post-activation potentiation, ultrasound imaging, conditioning activity, drop jump

This article is protected by copyright. All rights reserved. 


\section{Introduction}

In recent years it has become very popular to investigate various conditioning protocols, including heavy resistance and ballistic exercises, with the objective of enhancing subsequent athletic performance (DeRenne, 2010; Maloney et al., 2014). These performance gains are generally attributed to postactivation potentiation (PAP), a short-lasting mechanism that is thought to be of intramuscular origin, potentiating the muscle's maximum force output subsequent to conditioning contractions (Tillin \& Bishop, 2009). A number of additional mechanisms have been proposed, but clear evidence for their significant contributions is still lacking (Hodgson et al., 2005). Jumps, including drop jumps (DJ) and countermovement jumps, are commonly used to assess performance enhancement in the lower limbs. Various conditioning protocols that increase jump height can be found in the literature (e.g. see Hodgson et al., 2005; Maloney et al., 2014). Interestingly, there is poor evidence for the transfer of performance enhancement to other movements such as sprinting (Kümmel et al., 2016) or sprint cycling (French et al., 2003). Identifying the mechanisms that substantially contribute to potentiated jump performance would help to advance the implementation of jumping protocols in appropriate athletic disciplines. Maloney et al. (2014) proposed that conditioning activities might increase stiffness at the musculotendinous level during the jump, and thus allow a higher contribution of passive tension to overall force production. However, there is a lack of evidence for such an improved SSC efficacy following a conditioning activity. Consequently, the purpose of the present study was to examine muscletendon unit (MTU) behaviour during conditioned stretch-shortening cycle (SSC) exercise.

We hypothesized that conditioning leading to potentiated performance in SSC exercise would be accompanied by a higher triceps surae force, resulting in a larger Achilles tendon strain, and in turn enabling a larger amount of energy to be absorbed during the eccentric phase of

This article is protected by copyright. All rights reserved. 
the SSC. Furthermore, it was assumed that this would enhance SSC efficacy, resulting in higher mechanical work output of the MTU during a reactive movement.

\section{Methods}

\section{Subjects}

A total of 32 participants volunteered to participate in this study after giving their written informed consent; 18 in experiment 1 ( 5 female, 13 male; age: $25 \pm 3$ years; $71.3 \pm 9.7 \mathrm{~kg}$; $173 \pm 8 \mathrm{~cm}$ ), and 14 participants in experiment 2 ( 2 female, 12 male; age: $26 \pm 4$ years; 79.6 $\pm 9.2 \mathrm{~kg} ; 172 \pm 8 \mathrm{~cm})$. All study participants were healthy physically active sport students, who were familiar with the drop jump procedure and free from any pain. The study was approved by the local ethics committees of the University of Jyväskylä (Jyväskylä, Finland) and the University of Konstanz (Konstanz Germany) and conducted in accordance with the Declaration of Helsinki.

\section{Experimental design}

The study consisted of two similar experiments conducted in two different laboratories, with a small difference in the measurement set up. In both experiments, participants performed a total of 6 DJs from a drop height of $46 \mathrm{~cm}$. This height was chosen because there is evidence for the highest concentric peak power output to occur at drop heights between 40 to $60 \mathrm{~cm}$ (Walsh et al., 2004). Three DJs were performed immediately after 10 maximal repetitive reactive hops per DJ (HOP), whereas the other 3 DJs served as control (CTRL) without any prior conditioning. We chose repetitive hops as a conditioning activity, as previous studies have shown that they induce PAP in the triceps surae muscle and considerable performance enhancement in subsequent DJs (Bergmann et al., 2013; Kümmel et al., 2016). The order of the DJs was counterbalanced between all subjects. There was a break of 1 min between DJs,

This article is protected by copyright. All rights reserved. 
and an additional break of $5 \mathrm{~min}$ between the HOP and CTRL conditions. Participants were instructed to maximize their rebound jump height whilst keeping the ground contact time as short as possible. The two-legged DJ was always initiated with the right leg. Although the subjects were already highly familiar with the jumping procedure, they were additionally familiarized with the correct jumping technique on a separate day. A short warm-up routine 5 min prior to both experiments (consisting of 10 unloaded squats and 10 submaximal hops) was used to prepare the subjects for the subsequent jumps.

\section{Kinematic and kinetic recordings}

In both experiments, a marker-based motion capture system (Vicon Nexus®, Oxford, UK) in combination with ground reaction force plates (AMTI®, Watertown, USA) was used to calculate jump height and maximal concentric power, as well as the net joint moments and power output around the ankle joint during DJs (Bobbert et al., 1986b; Winter, 2009). Eleven auto-reflective markers were placed on the following anatomical landmarks of the right leg and the torso: fifth metatarsal bone, first metatarsal bone, lateral malleolus, calcaneus, lateral shank, lateral knee joint center, lateral thigh, greater trochanter, anterior and posterior iliac crest and corpus sternum. The sampling frequency of the motion capture system was set to $250 \mathrm{~Hz}$. The analogue signals from the force plates were synchronized and recorded with the motion capture system at a sampling frequency of $1 \mathrm{kHz}$.

\section{Electromyographic recordings}

In both experiments, EMG activity was recorded from the medial gastrocnemius (GM) and soleus (SOL) muscles of the right leg. In the first experiment, bipolar surface electrodes with a 5-mm diameter and a 10-mm inter-electrode distance (Ambu Blue Sensor N, Ballerup, Denmark) were used. Amplified (500x) EMG signals were sampled with a frequency of 1

This article is protected by copyright. All rights reserved. 
kHz. The reference electrode was placed on the tibia shin. In the second experiment, wireless electrodes (Delsys, Trigno, Boston, USA, 909x amplified, DC-500 Hz, 160 dB/Dec.) with an 11-mm inter electrode distance and integrated reference electrodes sampled the EMG signals at $2 \mathrm{kHz}$. In both experiments, analogue EMG signals were synchronized and recorded with the motion capture system (Vicon, Oxford, UK) by means of an A/D converter. The skin preparation and EMG electrodes positioning were in accordance with the SENIAM recommendations (Merletti et al., 2016), although in experiment 1 the GM electrodes were placed slightly more laterally on the muscle midbelly to allow fixation of the ultrasound probe.

\section{Ultrasound measurements}

B-mode ultrasound was used in both experiments to assess differences in triceps surae muscle-tendon behaviour between the conditioned and control DJs. In experiment 1 , the ultrasound device ( $\alpha 10$, Aloka, Tokyo, Japan; 7.5-MHz probe, $60 \mathrm{~mm}$ field of view) was used to identify length changes in the fascicles of the GM muscle during the DJ. The ultrasound probe was fixed on the midbelly of the medial gastrocnemius: first, the medial and lateral boundaries of this muscle were determined and then the probe was fixed in between, with the main axis coinciding with the line from the GM origin at the medial epicondyle to its insertion at the Achilles tendon. Afterwards, this position was slightly adjusted to ensure a parallel alignment to the upper and deeper aponeuroses of GM, and if possible, of SOL as well. A tailor-made foam pad was used for probe fixation with elastic bandages in order to reduce probe movement relative to the muscle. A compromise between image resolution and sampling frequency allowed a scanning depth of $60 \mathrm{~mm}$. These settings made it possible to track the fascicles of SOL in 12 of the 18 subjects from experiment 1 . Fascicle length was defined for both GM and SOL as the distance between the superficial and deeper aponeuroses along the fascicle orientation (see Fig. 1). The pennation angle of GM was defined between

This article is protected by copyright. All rights reserved. 
the muscle's deeper aponeurosis and its fascicle orientation; for SOL it was defined between its superficial aponeurosis and the fascicle orientation. The ultrasound videos of each jump were sampled with a frequency of $142 \mathrm{~Hz}$ and stored on a hard disc for later off-line analysis. A rectangular pulse from the ultrasound device was used for time synchronization with other data sampled by the motion capture system. We used a Matlab-based software package for semi-automated tracking of muscle fascicles in B-mode ultrasound image sequences (UltraTrack V4.1, see Cronin et al., 2011; Farris \& Lichtwark, 2016) to determine fascicle length, shortening velocity ( $1^{\text {st }}$ derivation of fascicle length) and pennation angle of GM and SOL during the ground contact phase of the DJs. Data were up-sampled to $1 \mathrm{kHz}$ by means of cubic spline interpolation.

This ultrasound-based method uses 2D pictures to assess 3D muscle architecture. Thus, it cannot account for the 3D rotation that muscles undergo during contraction (Cronin \& Lichtwark, 2013), and probe movement relative to the operating axis of the muscle must be minimized in order to reduce unnecessary error (Klimstra et al., 2007). To account for this, three motion capture markers were fixed to the ultrasound probe, making it possible to determine the tilt of the probe relative to the operating axis of the muscle during the drop jumps. We observed a marginal average tilt of $1.9 \pm 1.7^{\circ}$ during the ground contact phase. In the second experiment we used ultrasound imaging (Echoblaster 128, Telemed, Vilnius, Lithuania; 96-element linear probe, $60 \mathrm{~mm}$ field of view, B-mode, $7 \mathrm{MHz}$ ) to determine length changes of the Achilles tendon during DJs. The ultrasound probe was fixed above the muscle-tendon junction (MTJ) between GM and the Achilles tendon, aligned along the operating axis of the MTU between the medial condyles of the knee joint and the insertion of the Achilles tendon at the calcaneus. Elastic bandages were used to fix the probe to the most proximal point that still allowed visualization of the most distal point of the muscle-tendon junction during upright standing. This procedure ensured that in almost every subject it was

This article is protected by copyright. All rights reserved. 
possible to track the MTJ's maximum movement in the proximal direction during DJs. However, in 3 subjects the maximum elongation of the Achilles tendon during the ground contact phase of DJs could not be determined because the MTJ left the proximal field of view. Hence, these 3 subjects were treated as dropouts and their data were not included in the analyses. The image sampling frequency was set to $80 \mathrm{~Hz}$ with a scanning depth of $50 \mathrm{~mm}$. Two motion capture markers were attached to the ultrasound probe in order to facilitate the assessment of the absolute Achilles tendon length during the post-processing procedure. One of these markers was attached to a point that coincided with the distal and one to the proximal image border of the scanned area. We used the UltraTrack software (V4.1) for manual tracking of the MTJ and synchronized the ultrasound images with the motion capture system via a rectangular pulse sent by the ultrasound system. Manual tracking was performed twice to estimate intra-rating reliability. The ICC revealed a value of 0.975 , which demonstrates a high inter-rating reliability for this manual approach.

\section{Post-processing}

The rectified EMG of $\mathrm{GM}\left(\mathrm{rEMG}_{\mathrm{GM}}\right)$ and $\mathrm{SOL}\left(\mathrm{rEMG}_{\mathrm{SOL}}\right)$ signals were integrated for the pre-activation phase during the $150 \mathrm{~ms}$ prior to ground contact, the braking phase (ground contact until the time point where the hip marker reached its lowest height during the jump), and the push-off phase (lowest point of the hip marker until take-off) for univariate group comparison between CTRL and HOP.

For comparison of the time-series, the rectified EMG was normalized to the mean EMG activity during the ground contact phase of the corresponding control jump. It has to be noted that in 6 subjects of experiment 1, motion artefacts were found in the EMG signal during the post-processing procedure, which were probably caused by the fixation of the ultrasound probe. These subjects' data were removed from the rEMG analyses (total remaining $\mathrm{N}=26$ ).

This article is protected by copyright. All rights reserved. 
Median EMG frequency of GM and SOL was calculated for the pre-activation and ground contact phases. In addition, a Fast Fourier Transformation (done with MATLAB ${ }^{\circledR}$ R2016a) was applied to the EMG signals, once with a length of $150 \mathrm{~ms}$ for the pre-activation phase and once over the duration of the ground contact phase. The resulting frequency spectra for the pre-activation and ground contact phases were then averaged for all participants.

In order to determine changes in the movement pattern between conditions, hip, knee, and ankle joint angles were estimated based on kinematic and kinetic data from the initial ground contact, the push-off phase (i.e. lowest point of the centre of mass during ground contact), and for the time point of the take-off when the right leg left the force plate. Joint angles were calculated according to the method of Bobbert et al. (1987).

MTU lengths ( $\mathrm{L}_{\mathrm{MTU}}$ ) of the GM and SOL were calculated by combining the ankle and knee joint angles for each jump with the equations of Hawkins and Hull (Hawkins \& Hull, 1990), and the first derivation of $\mathrm{L}_{\mathrm{MTU}}$ was used to calculate MTU shortening velocities ( $\dot{\mathrm{L}}_{\mathrm{MTU}}$ ). Moment arm and plantar flexion moment for the ankle joint were estimated following Bobbert et al. (1986b), and were then used to calculate the force acting on the Achilles tendon $\left(\mathrm{F}_{\text {tendon }}\right)$. The relative contributions of GM and SOL to total Achilles tendon force were assumed to be constant between all conditions, and these relative proportions were taken from their relative physiological cross-sectional areas among the triceps surae (GM: 15.4\% and SOL: 57\%), which have been reported by Fukunaga et al. (1996). The mechanical work $\left(\mathrm{W}_{\mathrm{MTU}}\right)$ done by the SOL and GM MTUs during the DJs was calculated by the numerical integration of MTU power in the eccentric and concentric phases of the DJs (Fukashiro et al., 2005; Fukashiro et al., 2006), see formula [1]:

$$
\mathrm{W}_{\mathrm{MTU}}=\int\left(\dot{\mathrm{L}}_{\mathrm{MTU}} * \mathrm{~F}_{\mathrm{MTU}}\right) \mathrm{dt} \quad[1]
$$

In addition, data obtained in experiment 1 were used to determine the lengths of the passive elastic structures ( $\mathrm{L}_{\text {tendon }}$ ) from the GM and SOL MTUs, which was done with a geometrical

This article is protected by copyright. All rights reserved. 
planimetric muscle-tendon model (Allinger \& Herzog, 1993). In this model, the muscle's pennation angle (PEN), fascicle length (FASC) and estimated MTU length were used to calculate tendon length, see formula [2]:

$$
\mathrm{L}_{\text {tendon }}=\mathrm{L}_{\mathrm{MTU}}-(\mathrm{FASC} * \mathrm{PEN}) \quad[2] \text {. }
$$

The ultrasound data of experiment 2 were used to determine GM Achilles tendon length ( $\mathrm{L}_{\text {Achilles GM}}$ ) during each DJ by adopting a similar approach to that used by Lichtwark \& Wilson (2005) during one-legged hopping. The measured tendon length $\left(\mathrm{L}_{\text {meas }}\right)$ was determined as the distance between the distal image border and the manually tracked MTJ along the orientation of the tendon in the ultrasound images (see Fig. 2 A). Absolute Achilles tendon length ( $\mathrm{L}_{\text {Achilles } \mathrm{GM}}$ ) was then calculated as the sum of $\mathrm{L}_{\text {meas }}$ and the length projection of the vector between the calcaneus and the distal probe marker along the operating axis of the MTU ( $\mathrm{L}_{\text {calc }}$; Fig. 2 B). The probe movement along the shank was determined by the projection of its motion capture markers to the longitudinal axis of the shank, which was spanned by one marker attached to the lateral malleoli and one around the middle of the shank (Fig. 2 C). Instantaneous changes in the distance between the projected markers and the lateral malleoli were calculated $\left(\mathrm{d}_{\mathrm{dist}} \& \mathrm{~d}_{\text {prox }}\right)$. The instantaneous changes between both markers were then subtracted from the absolute Achilles tendon length, resulting in the following formula for estimated $\mathrm{L}_{\text {Achilles } \mathrm{GM} \text { : }}$

$$
\mathrm{L}_{\text {Achilles GM }}=\mathrm{L}_{\text {meas }}+\mathrm{L}_{\text {calc }}-\left[\frac{\Delta \mathrm{d}_{\text {dist }}+\Delta \mathrm{d}_{\text {prox }}}{2}\right] \quad[3] \text {. }
$$

All variables were calculated for the right leg only.

\section{Statistics}

This article is protected by copyright. All rights reserved. 
For each subject and each condition, the jump with the highest rebound jump height was selected and used for statistical comparison.

Kolmogorov-Smirnov tests were performed for each of the analysed variables, revealing no significant deviations from the normal distribution. Paired $t$ tests were used for statistical comparison of scalar variables between HOP and CTRL. One-dimensional statistical parametric mapping (SPM) was used to compare the muscle activity pattern, fascicle length, pennation angle, tendon length, and inversely calculated mechanical variables between both conditions. SPM allows the calculation of $t$ statistics for time-normalized scalar fields $(\operatorname{SPM}\{\mathrm{t}\})$. The corrected $t$ distribution is computed by means of both random field theory (RFT; Adler \& Taylor, 2007) and the level of significance $(\alpha=0.05)$. RFT reveals the critical threshold $t^{*}$ of $\operatorname{SPM}\{\mathrm{t}\}$ based on the field smoothness estimated from temporal derivatives over the entire normalized time-series. For clusters of the $\operatorname{SPM}\{\mathrm{t}\}$ trajectory that exceed $t^{*}$, the null hypothesis is rejected. Time-normalization of the scalar fields was done for the whole ground contact phase of each jump. The statistical approach of $\operatorname{SPM}\{t\}$ in this study is conceptually identical to the univariate paired $t$ test for scalar variables. A detailed description can be found elsewhere (Pataky, 2010; Pataky et al., 2013). All SPM $\{\mathrm{t}\}$ calculations were done in Matlab, based on the code provided by Pataky (2012).

Univariate paired student's $t$ tests were used to compare performance variables of the jumps (jump height, ground reaction force, peak concentric power, ground contact time, joint angles) between CTRL and HOP. In addition, we used a one-way analysis of variance with repeated measures (rmANOVA) to test the effect of conditioning hops on the total amount of work done by the GM and SOL MTUs, as well as the work derived from the Achilles tendon strain energy during the eccentric and concentric phases of the DJs. The same procedure was used to test for statistical differences in GM and SOL muscle activity (integrated rEMG and median frequency) during the pre-activation, braking and push-off phases of the conditioned

This article is protected by copyright. All rights reserved. 
and control DJs. In case of significant differences $(\mathrm{p}<0.05)$, Bonferroni corrected post hoc analyses were carried out. Effect sizes $\left(\mathrm{d}_{\mathrm{z}}\right)$ were estimated according to the calculation of Cohen's $d$ for dependent means.

\section{Results}

In experiment 1 , jump height after 10 conditioning hops increased by $12 \%(\mathrm{p}<0.001$, $\mathrm{d}_{\mathrm{z}}=0.62$ ), which was accompanied by a significant $8 \%$ increase in concentric peak power $\left(\mathrm{P}_{\text {peak }} ; \mathrm{p}<0.01, \mathrm{~d}_{\mathrm{z}}=0.35\right)$. Similar results were observed in the second experiment; hops increased jump height by $19 \%\left(\mathrm{p}<0.001, \mathrm{~d}_{\mathrm{z}}=0.98\right)$ and $\mathrm{P}_{\text {peak }}$ by $13 \%\left(\mathrm{p}<0.05, \mathrm{~d}_{\mathrm{z}}=0.61\right)$. In both experiments ground reaction forces $\left(\mathrm{F}_{\max }\right)$ and ground contact times (CT) did not statistically differ between HOP and CTRL (see Supporting Information Table S 1).

No statistical differences were observed between HOP and CTRL in joint angles at the time of initial ground contact or at take-off (see Supporting Information Table S 2). However, during the initial push-off phase, the knee and ankle joint angles were significantly smaller during HOP compared to CTRL, i.e. there was an increase in movement amplitude at both joints (knee: $-3.8^{\circ}, \mathrm{p}<0.01, \mathrm{~d}_{\mathrm{z}}=0.45 ;$ ankle: $\left.-2.8^{\circ}, \mathrm{p}<0.001, \mathrm{~d}_{\mathrm{z}}=0.46\right)$.

GM fascicle length $\left(\mathrm{FASC}_{\mathrm{GM}}\right)$ and $\mathrm{SOL}$ fascicle length $\left(\mathrm{FASC}_{\mathrm{SOL}}\right)$ exhibited a characteristic SSC behaviour during ground contact (Fig. 2). $\operatorname{SPM}\{t\}$ revealed that hops prior to DJs significantly reduced the lengthening of the $\mathrm{FASC}_{\mathrm{GM}}$, indicated by a $4-5 \mathrm{~mm}$ reduction in fascicle length during 42-65 \% of the CT for HOP compared to CTRL (18 subjects, p<0.01,Fig. 3). For SOL, we were able to track FASC $_{S O L}$ in 12 subjects but did not observe a significant difference between HOP and CTRL (Fig. 3). SPM $\{t\}$ showed no statistical differences between HOP and CTRL in pennation angle in GM or SOL (see Supporting Information Fig. S1). The calculated length of the GM passive elastic structures revealed a significant elongation of $4-5 \mathrm{~mm}$ between $40-70 \%$ of ground contact for HOP compared to

This article is protected by copyright. All rights reserved. 
CTRL ( $\mathrm{p}<0.001$,Fig. 4). In contrast, no changes were observed in SOL passive elastic structures between HOP and CTRL (Fig. 4).

The changes at the level of the MTU in the conditioned DJs were accompanied by a significantly higher force acting on the triceps surae muscle $\left(\mathrm{F}_{\text {tendon }}\right)$ at $40-45 \%$ of CT $(+300$ $\mathrm{N}, \mathrm{p}<0.001$, Fig. 5 A). This led to a significant increase in negative work done by the GM and SOL MTUs during the eccentric phase $(\mathrm{GM}:+14 \%$, SOL: $+11 \%, \mathrm{p}<0.05$ for both, GM: $d_{z}=0.45$, SOL: $d_{z}=0.53$, Fig. 5 B), and a significantly higher amount of positive work done during the concentric phase of the DJ for HOP compared to CTRL (GM: +9 \%, SOL: $+12 \%$, $\mathrm{p}<0.05$ for both, GM: $\mathrm{d}_{\mathrm{z}}=0.37$, SOL: $\mathrm{d}_{\mathrm{z}}=0.71$, Fig. 5 B). Note that negative work denotes a higher amount of absorbed energy (detailed values can be found in the Supporting Information Table S 3).

In experiment 2 the $\operatorname{SPM}\{t\}$ revealed a significantly longer $\mathrm{L}_{\text {Achilles } \mathrm{GM}}$ during $47-90 \%$ of $\mathrm{CT}$ for HOP compared to CTRL ( $\mathrm{p}<0.001$, Fig. 6). This difference ranged between $3-5 \mathrm{~mm}$. Concomitantly, the inversely calculated $\mathrm{F}_{\text {tendon }}$ was significantly higher, by up to $500 \mathrm{~N}$, during 51-92\% of $\mathrm{CT}$ for the HOP condition $(\mathrm{p}<0.001, \mathrm{p}<0.05$, Fig. 6). This difference contributed to a significant difference between conditions in the power of the medial gastrocnemius MTU ( $\left.\mathrm{P}_{\mathrm{MTU}}\right)$. HOP decreased $\mathrm{P}_{\mathrm{MTU}}$ by 30-50 Watts during 40-45 \% of CT $(\mathrm{p}<0.001)$, whereas during the late concentric phase of the DJ $\left(88-91 \%\right.$ of CT), $\mathrm{P}_{\mathrm{MTU}}$ increased significantly by $25-30$ Watts compared to CTRL ( $<<0.05$, Fig. 6). Expressed as the total amount of work done by the GM MTU - or more precisely derived from the Achilles tendon strain energy ( Work $_{\text {Achilles GM }}$ ) - these changes culminated in a significant difference between HOP and CTRL (Fig. 7). HOP resulted in significantly higher negative Work $_{\text {Achilles }}$ GM for the eccentric phase $\left(-28 \%, \mathrm{p}<0.01\right.$; CTRL: $-21.1 \pm 9.5 \mathrm{~J}$; HOP: $\left.26.9 \pm 9.7 \mathrm{~J}, \mathrm{~d}_{\mathrm{z}}=0.60\right)$ but significantly higher energy expenditure during the concentric phase compared to CTRL $\left(+33 \%, p<0.01\right.$; CTRL: $8.3 \pm 2.9 \mathrm{~J} ;$ HOP: $\left.11.1 \pm 3.0 \mathrm{~J}, \mathrm{~d}_{\mathrm{z}}=0.95\right)$.

This article is protected by copyright. All rights reserved. 
SPM analysis of the rEMG curves for GM and SOL revealed no statistical differences between HOP and CTRL during the pre-activation phase or during the ground contact phase of the jumps performed in experiment 1 (see Supporting Information Fig. S 2). These results were confirmed by the absence of any significant differences between CTRL and HOP in integrated EMG, median frequency and power spectra in both muscles (see Supporting Information Table S 4 and 5).

\section{Discussion}

In the present study ten repetitive reactive hops were used to potentiate subsequent DJ performance in order to investigate the impact of the conditioning activity on triceps surae SSC performance as well as the underlying mechanisms. Our results showed that the hops increased subsequent DJ performance as indicated in both experiments by the superior jump height and concentric peak power. These findings are in line with previous studies that used repetitive reactive hops to potentiate subsequent DJ performance (Bergmann et al., 2013; Kümmel et al., 2016).

This potentiated performance was accompanied by altered mechanical behaviour of the medial gastrocnemius MTU. We observed a reduced fascicle lengthening in the transition from MTU lengthening to shortening, resulting in an increased elongation of the passive elastic structures of the MTU, such as the Achilles tendon or the connective tissue within the muscle.

\section{Efficacy of SSC function}

From a biomechanical point of view, the changes observed in GM strongly suggest that the conditioning hops improve the SSC function of storing and reusing elastic energy in the tendinous structures during a subsequent drop jump. The calculated values of the mechanical

This article is protected by copyright. All rights reserved. 
work done by GM and SOL demonstrated that for both MTUs, the amount of energy that was absorbed during the braking phase and released during the subsequent upward acceleration was higher when conditioning hops were performed prior to DJs. The second experiment indicated that at least part of the increased mechanical work derived from an enhanced stretch and recoil of the Achilles tendon. Even though the measures of fascicle length and the estimation of Achilles tendon length were done in two distinct experiments, the conjunction of both observations substantiates the notion that conditioning hops increase triceps surae (and thus Achilles tendon) force, enabling more energy to be converted into mechanical work (Kawakami et al., 2002). Several points support this assumption: (a) the calculated length of the GM passive elastic structures in experiment 1 revealed a significant elongation for the HOP condition, (b) in both experiments a significantly higher force was found to act on the Achilles tendon $\left(\mathrm{F}_{\text {tendon }}\right)$ in the HOP condition (c.f. Fig. 5 A and Fig. 6), and (c) the shortened FASC $_{\mathrm{GM}}(40-70 \%$ of CT) coincided chronologically with the development of an elongated $\mathrm{L}_{\text {Achilles GM }}$ (47-90 \% of CT; c.f. Fig. 3 and Fig. 6).

No difference was found in the behaviour of FASC $_{\mathrm{SOL}}$ between HOP and CTRL. Regarding the amount of calculated MTU work, a similar response to that of GM occurred in SOL when DJs were performed with prior conditioning: more energy was transferred from the eccentric to the concentric phase of the jump (Fig. 5 B). The underlying mechanism for this enhanced performance becomes obvious when the fascicle behaviour is considered with respect to the force acting on the MTU. The increase in $\mathrm{F}_{\text {tendon }}$ (Fig. 5 A, Fig. 6) in conjunction with the absence of any changes at the level of the fascicles could indicate that even SOL muscle stiffness was higher in the conditioned DJ. However, no significant change was observed in the calculated length of the series elastic elements of the SOL muscle, which raises the question of whether the relative contribution to $F_{\text {tendon }}$ among the muscles changed after conditioning.

This article is protected by copyright. All rights reserved. 
The GM is a bi-articular muscle, which is thought to transfer energy and power during human locomotion from one joint to another (G. v. van Ingen Schenau et al., 1987; G. J. van Ingen Schenau et al., 1990). Bobbert et al. (1986a) deduced from their biomechanical model of onelegged jumps that the power transferred by the gastrocnemius muscle from the knee to the ankle joint was more than 2-fold higher than the power supplied by the fibres and the tendons of the triceps surae muscle group. Forward dynamic simulations revealed that maximum jump height critically depends on the combination of moment arms of the gastrocnemius muscle at the knee and ankle joints (Bobbert \& van Zandwijk, 1994). In the present study, the small changes observed in knee and ankle angles during the initial push-off phase might have affected the mechanics of the GM (Visser et al., 1990) and facilitated its function in transferring power from knee extension to plantar flexion. If so, the force acting on GM might have increased, resulting in a change in GM muscle-tendon interaction, whereas the SOL remained unaffected. This in turn could explain the different behaviour of the GM and SOL fascicles during the conditioned DJs and would be in line with the findings of Sousa et al. (2007). The latter authors found that with increasing DJ height the fascicle behaviour of GM changed from a shortening to a lengthening pattern during the braking phase, whereas the fascicle behaviour of SOL remained unchanged. These results may indicate a change in the relative force contribution of GM and SOL to $\mathrm{F}_{\text {tendon }}$ with increasing drop height.

This has some consequences for the calculated work of the SOL and GM MTUs in the present study. The reported values for GM might be underestimated, since the force acting on this part of the muscle might be higher due to its bi-articular nature, and thus its role in energy transfer between joints. As SOL acts parallel to GM, the calculated work done by SOL might thus be overestimated. However, if the relative force contribution between GM and SOL changed in favour of higher GM force after the conditioning hops, this would result in higher GM muscle stiffness, whereas SOL stiffness may not change. Nonetheless, one

This article is protected by copyright. All rights reserved. 
could infer that conditioning hops improve the efficacy of SSC function in GM in a subsequent DJ by increasing its muscle force during ground contact, with the contribution of SOL remaining elusive. The different fascicle behaviour between GM and SOL observed for the conditioned DJ in the present study is likely attributable to functional differences between these muscles during drop jumping (e.g. Sousa et al., 2007).

\section{Mechanisms of increased muscle force}

An obvious question arises: where does the observed increase in muscle force during the conditioned jumps originate from? Previous studies that investigated the fascicle behaviour of the leg extensors during drop jumps observed that with increasing drop height and vertical ground reaction force, the vastus lateralis (Ishikawa \& Komi, 2004) and medial gastrocnemius muscles (Sousa et al., 2007) exhibited reduced lengthening during the eccentric braking phase. On the contrary, the soleus fascicles showed no changes in stretchshortening amplitudes at varying drop heights (Sousa et al., 2007). These fascicle behaviours, especially for GM and SOL, are similar to the length changes that were found in the present

study. However, the results of the previous experiments were accompanied by increased EMG activity in the investigated muscles during the pre-activation and braking phases, implying that higher pre-activity and activity during the braking phase in the extensor muscles is necessary to increase muscle and thus leg stiffness during a DJ (Arampatzis et al., 2001). The absence of any significant differences in integrated EMG between CTRL and HOP in the pre-activation and braking phases, in the continuous activity of GM and SOL, and in the median frequency and power spectra of each muscle indicate that this was not the case for the augmented muscle force observed in the present study. In fact, similar results were reported in the study of Bergmann et al. (2013). The authors presented evidence that the potentiated DJ performance subsequent to conditioning hops was not associated with changes

This article is protected by copyright. All rights reserved. 
in the activation pattern of the major leg muscles. In contrast they found a significant positive correlation between potentiated twitch force of the triceps surae and potentiated jump height when repetitive hops were performed prior to both measures. This corroborates the theory that changes in intrinsic muscle properties rather than neuronal effects contribute to the increased muscle force during conditioned DJs. Indeed, one explanation might come from the underlying mechanisms of the PAP effect; it is generally accepted that heavy muscle contractions increase the phosphorylation of regulatory light chains within the actin-myosin complex, resulting in a higher force-generating capability (for review see Tillin \& Bishop, 2009). Thus, the muscle can produce more force in response to a given action potential, as evidenced for example by potentiated electrically-evoked muscle twitches subsequent to conditioning. As PAP appears in concentric, isometric and eccentric muscle contractions (Babault et al., 2008), we propose that the same mechanism might have caused the increase in muscle force in the conditioned DJs. It has been consistently reported that 10 repetitive hops induce PAP in the triceps surae muscle (Bergmann et al., 2013). We assume that PAP allows the muscle to produce a higher force within a shorter time period in response to a similar neural input resulting in a higher stiffness. Given the fact that repetitive hops do not alter Achilles tendon stiffness (Peltonen et al., 2010), this increase in muscle stiffness results in a higher force that acts on the whole GM muscle-tendon unit and thus elongates its passive elastic structures to a greater extent. However, the effects of conditioning on SOL function are rather unclear for the above-mentioned reasons. If PAP is responsible for the increased stiffness, a smaller effect would be expected in SOL compared to GM. PAP has been shown to have a larger effect on muscles that contain a higher percentage of fast-twitch fibres (Hamada et al., 2000, 2003), and SOL has a lower percentage of fast twitch fibres than GM (Gollnick et al., 1974). It is possible that the conditioning hops induced a different degree of PAP between the SOL and GM muscles (i.e. caused by different fibre type compositions),

This article is protected by copyright. All rights reserved. 
which might have contributed to divergent patterns of muscle-tendon interaction during the conditioned DJs.

Despite several limitations (see section below), we emphasize that the major indications for the abovementioned cascade could be observed in the first experiment of the present study: reduced fascicle lengthening (between -4 and $-5 \mathrm{~mm}, 42-65 \%$ of CT) and simultaneously elongated length of passive elastic structures (between 4 and $5 \mathrm{~mm}, 40-70 \% \mathrm{GT}$ ) of the GM coincided with increased maximal force acting on the triceps surae muscle (up to $300 \mathrm{~N}$, 40$45 \%$ of CT). Hence, it seems appropriate to conclude that conditioning hops increase the efficacy of SSC function due initially to increased muscle force. Moreover it appears that this results from intrinsic mechanisms within the triceps surae, such as the PAP effect.

\section{Limitations}

We calculated MTU work by separating the Achilles tendon force into constant relative contributions of GM and SOL (Fukunaga et al., 1996). As the relative force contribution of GM and SOL to $F_{\text {tendon }}$ doesn't necessarily stay constant throughout the DJ we could have over- or underestimated GM forces. However, it should be noted that fascicle length, pennation angle and overall MTU length did not differ between HOP and CTRL in SOL indicating an unchanged length of the passive elastic SOL structures. Thus, we assume that a potential shift could have only increased the relative force contribution of GM which would have further increased Achilles tendon strain energy of GM.

Triceps surae and Achilles tendon forces were calculated using the resultant net moment around the ankle joint. Other sources (e.g. ligaments, other synergists and antagonists) that contribute to the ankle joint moment were not considered in this calculation. Therefore the calculated force values for the triceps surae muscle and Achilles tendon are overestimated. However, due to the repeated measures study design we assumed the resultant calculation

This article is protected by copyright. All rights reserved. 
error to be constant between both conditions, negating its influence on the statistical difference between CTRL and HOP.

There are also some limitations of the Achilles tendon length estimation in experiment 2. As only one ultrasound probe was available, the distal insertion point of the tendon was assumed to be at the calcaneus, which was represented by a marker on the skin that was tracked via motion capture. Thus the calculated length may include some movement of the skin where the marker was attached. In addition, it is well known that the Achilles tendon follows a curved shape between its distal and proximal insertion points (Arampatzis et al., 2008; Stosic \& Finni, 2011), which might lead to an overestimation of tendon elongation. Hence, the reported tendon length of the present study might not represent the actual absolute value. However, due to the repeated measures study design, it can be argued that both the effect of skin movement under the calcaneus marker and the tendon shape had similar effects on the HOP and CTRL conditions, so that it had probably little influence on the main results, i.e., the comparison between the two conditions.

Finally, it is important to note that the observed changes in muscle-tendon interaction during a conditioned DJ refer to the right leg only. Although it seems likely that the left leg behaved in a similar way, the increase in work done by the GM and SOL MTUs still cannot completely explain the increase in jump height $(3.1 \mathrm{~cm}$ for experiment 1 and $4.9 \mathrm{~cm}$ for experiment 2). For example, the additional work done by the GM and SOL MTUs in experiment 1 corresponds to an additional jump height of $\sim 1 \mathrm{~cm}$, whereas the extra work derived from the strain energy of the Achilles tendon in experiment 2 accounts for an average increase in height of only $\sim 0.5 \mathrm{~cm}$. One might speculate that either a change in the relative force contribution between the MTUs of the triceps surae underestimates the amount of extra work derived from Achilles tendon strain energy, or other muscles that also undergo a SSC

This article is protected by copyright. All rights reserved. 
during DJs such as the quadriceps femoris might also benefit from conditioning hops and contribute to subsequent potentiated performance.

\section{Conclusions}

We conclude that 10 conditioning hops increase subsequent drop jump performance, in part due to additional mechanical work done by the MTU of the triceps surae. Our ultrasound imaging results provide strong evidence for an increased force of GM as a contributor to this potentiated performance via a more effective stretch-shortening cycle. Moreover, the absence of any changes in EMG suggests that other mechanisms such as altered intrinsic muscle properties may contribute to modulated fascicle behaviour during conditioned DJs.

\section{Perspective}

The results of the present study have potential for transfer to athletic performance, especially in movements that include the stretch-shortening cycle, i.e. energy storage and release in the triceps surae. Incorporating the major finding of the present study, that it is most probably the increase in triceps surae muscle force that accounts for power potentiation, one should identify athletic tasks where maximum forces acting on the tendon during the SSC make muscle stiffness a limiting factor for performance.

Acknowledgement: The authors thank Basílio Gonçalves, Kim Broscheid, Minttu Virtanen, Marcello Grassi, Felix Thomas, and Benjamin Weiß for their help with experiment preparation and data acquisition.

This work was supported by a travel grant from the Excellence Initiative of the University of Konstanz (Germany). The authors declare no conflict of interest.

This article is protected by copyright. All rights reserved. 


\section{References}

Adler, R. J., \& Taylor, J. E. (2007). Random Fields and Geometry. New York: SpringerVerlag.

Allinger, T., \& Herzog, W. (1993). Calculated fiber lengths in cat gastrocnemius muscle during walking. J Biomech, 26(3), 296.

Arampatzis, A., De Monte, G., \& Karamanidis, K. (2008). Effect of joint rotation correction when measuring elongation of the gastrocnemius medialis tendon and aponeurosis. Journal of Electromyography and Kinesiology, 18(3), 503-508.

Arampatzis, A., Schade, F., Walsh, M., \& Brüggemann, G.-P. (2001). Influence of leg stiffness and its effect on myodynamic jumping performance. J Electromyogr Kines, 11(5), 355-364.

Babault, N., Maffiuletti, N. A., \& Pousson, M. (2008). Postactivation potentiation in human knee extensors during dynamic passive movements. Med Sci Sports Exerc, 40(4), 735-743.

Bergmann, J., Kramer, A., \& Gruber, M. (2013). Repetitive Hops Induce Postactivation Potentiation in Triceps Surae as well as an Increase in the Jump Height of Subsequent Maximal Drop Jumps. PloS one, 8(10), e77705.

Bobbert, M. F., Huijing, P. A., \& van Ingen Schenau, G. (1987). Drop jumping. I. The influence of jumping technique on the biomechanics of jumping. Med Sci Sports Exerc, 19(4), 332-338.

Bobbert, M. F., Huijing, P. A., \& van Ingen Schenau, G. J. (1986a). An estimation of power output and work done by the human triceps surae musle-tendon complex in jumping. Journal of biomechanics, 19(11), 899-906.

Bobbert, M. F., Huijing, P. A., \& van Ingen Schenau, G. J. (1986b). A model of the human triceps surae muscle-tendon complex applied to jumping. J Biomech, 19(11), 887898.

Bobbert, M. F., \& van Zandwijk, J. P. (1994). Dependence of human maximum jump height on moment arms of the bi-articular m. gastrocnemius; a simulation study. Human Movement Science, 13(5), 697-716.

Cronin, N. J., Carty, C. P., Barrett, R. S., \& Lichtwark, G. (2011). Automatic tracking of medial gastrocnemius fascicle length during human locomotion. J Appl Physiol, 111(5), 1491-1496.

Cronin, N. J., \& Lichtwark, G. (2013). The use of ultrasound to study muscle-tendon function in human posture and locomotion. Gait Posture, 37(3), 305-312.

DeRenne, C. (2010). Effects of postactivation potentiation warm-up in male and female sport performances: A brief review. Strength Cond, 32(6), 58-64.

Farris, D. J., \& Lichtwark, G. A. (2016). UltraTrack: Software for semi-automated tracking of muscle fascicles in sequences of B-mode ultrasound images. Comput Methods Programs Biomed, 128, 111-118.

French, D. N., Kraemer, W. J., \& Cooke, C. B. (2003). Changes in dynamic exercise performance following a sequence of preconditioning isometric muscle actions. $J$ Strength Cond Res, 17(4), 678-685.

Fukashiro, S., Hay, C., \& Nagano, A. (2006). Biomechanical behavior of muscle-tendon complex during dynamic human movements. J App Biomech, 22(2), 131.

Fukashiro, S., Kurokawa, S., Hay, D. C., \& Nagano, A. (2005). Comparison of muscletendon interaction of human m. gastrocnemius between ankle-and drop-jumping. $J$ Sport Health Sci, 3, 253-263.

Fukunaga, T., Roy, R. R., Shellock, F. G., Hodgson, J. A., \& Edgerton, V. R. (1996). Specific tension of human plantar flexors and dorsiflexors. J Appl Physiol, 80(1), 158-165.

This article is protected by copyright. All rights reserved. 
Gollnick, P. D., Sjödin, B., Karlsson, J., Jansson, E., \& Saltin, B. (1974). Human soleus muscle: a comparison of fiber composition and enzyme activities with other leg muscles. Pflügers Archiv, 348(3), 247-255.

Hamada, T., Sale, D. G., MacDougall, J. D., \& Tarnopolsky, M. A. (2000). Postactivation potentiation, fiber type, and twitch contraction time in human knee extensor muscles. Journal of Applied Physiology, 88(6), 2131-2137.

Hamada, T., Sale, D. G., MacDougall, J. D., \& Tarnopolsky, M. A. (2003). Interaction of fibre type, potentiation and fatigue in human knee extensor muscles. Acta Physiologica Scandinavica, 178(2), 165-173.

Hawkins, D., \& Hull, M. (1990). A method for determining lower extremity muscle-tendon lengths during flexion/extension movements. J Biomech, 23(5), 487-494.

Hodgson, M., Docherty, D., \& Robbins, D. (2005). Post-activation potentiation. Sports Med, 35(7), 585-595.

Ishikawa, M., \& Komi, P. V. (2004). Effects of different dropping intensities on fascicle and tendinous tissue behavior during stretch-shortening cycle exercise. J App Physiol, 96(3), 848-852.

Kawakami, Y., Muraoka, T., Ito, S., Kanehisa, H., \& Fukunaga, T. (2002). In vivo muscle fibre behaviour during counter - movement exercise in humans reveals a significant role for tendon elasticity. $J$ Physiol (Lond), 540(2), 635-646.

Klimstra, M., Dowling, J., Durkin, J. L., \& MacDonald, M. (2007). The effect of ultrasound probe orientation on muscle architecture measurement. J Electromyogr Kines, 17(4), 504-514.

Kümmel, J., Bergmann, J., Prieske, O., Kramer, A., Granacher, U., \& Gruber, M. (2016). Effects of conditioning hops on drop jump and sprint performance: a randomized crossover pilot study in elite athletes. BMC Sports Sci Med Rehabil, 8, 1. doi:10.1186/s13102-016-0027-z

Lichtwark, G. A., \& Wilson, A. (2005). In vivo mechanical properties of the human Achilles tendon during one-legged hopping. Journal of Experimental Biology, 208(24), 47154725.

Maloney, S. J., Turner, A. N., \& Fletcher, I. M. (2014). Ballistic Exercise as a Pre-Activation Stimulus: A Review of the Literature and Practical Applications. Sports Med, 44(10), 1347-1359.

Merletti, R., Rau, G., Disselhorst-Klug, C., Stegeman, D., \& Hägg, G. (2016). Surface ElectroMyoGraphy for the Non-Invasive Assessment of Muscles (SENIAM). Retrieved from http://www.seniam.org/

Pataky, T. C. (2010). Generalized n-dimensional biomechanical field analysis using statistical parametric mapping. J Biomech, 43(10), 1976-1982.

Pataky, T. C. (2012). One-dimensional statistical parametric mapping in Python. Comput Methods Biomech Biomed Eng, 15(3), 295-301.

Pataky, T. C., Robinson, M. A., \& Vanrenterghem, J. (2013). Vector field statistical analysis of kinematic and force trajectories. J Biomech, 46(14), 2394-2401.

Peltonen, J., Cronin, N. J., Avela, J., \& Finni, T. (2010). In vivo mechanical response of human Achilles tendon to a single bout of hopping exercise. J Exp Biol, 213(8), 12591265.

Sousa, F., Ishikawa, M., Vilas-Boas, J., \& Komi, P. V. (2007). Intensity-and muscle-specific fascicle behavior during human drop jumps. J Appl Physiol, 102(1), 382-389.

Stosic, J., \& Finni, T. (2011). Gastrocnemius tendon length and strain are different when assessed using straight or curved tendon model. European journal of applied physiology, 111(12), 3151-3154.

This article is protected by copyright. All rights reserved. 
Tillin, M. N. A., \& Bishop, D. (2009). Factors modulating post-activation potentiation and its effect on performance of subsequent explosive activities. Sports Med, 39(2), 147-166.

van Ingen Schenau, G. J., Bobbert, M. F., \& van Soest, A. J. (1990). The unique action of biarticular muscles in leg extensions Multiple muscle systems (pp. 639-652): Springer.

van Ingen Schenau, G. v., Bobbert, M., \& Rozendal, R. (1987). The unique action of biarticular muscles in complex movements. Journal of Anatomy, 155, 1.

Visser, J., Hoogkamer, J., Bobbert, M., \& Huijing, P. (1990). Length and moment arm of human leg muscles as a function of knee and hip-joint angles. European journal of applied physiology and occupational physiology, 61(5-6), 453-460.

Walsh, M., Arampatzis, A., Schade, F., \& Brüggemann, G. (2004). The effect of drop jump starting height and contact time on power, work performed, and moment of force. $J$ Strength Cond Res, 18(3), 561-566.

Winter, D. A. (2009). Kinetics: forces and moments of force: Wiley Online Library.

\section{Figure Captions}

Fig. 1 Left: Fascicle length tracking of the ultrasound images of the medial gastrocnemius (GM, upper dotted box) and soleus (SOL, lower dotted box) between the superficial and deeper aponeuroses for each muscle during drop jumps. Note: the contrast has been reduced in this picture for better perceptibility of the tracked fascicles (solid lines).

Right: Tracking of the muscle tendon junction (MTJ) between the GM and the proximal Achilles tendon (solid lines). The upper and lower pictures denote the amplitude of MTJ movement during a drop jump. Note: total Achilles tendon length was calculated by adding the actual tracked length to the distance between the distal end of the ultrasound probe and the Achilles tendon insertion on the calcaneus.

Fig. 2 A: The distance between the ultrasound probe and Achilles tendon was estimated with the help of motion capture markers attached to the probe and to the calcaneus. This distance was projected to the instantaneous working axis of the medial gastrocnemius (GM) between its origin and insertion. B: This projection revealed the calculated part of the Achilles tendon length $\left(\mathrm{L}_{\text {calc }}\right)$, which was added to the measured length of the tendon $\left(\mathrm{L}_{\text {meas }}\right)$ via tracking of the muscle tendon junction (MTJ). C: $\mathrm{L}_{\text {meas }}$ was corrected for the proximal $\left(\mathrm{d}_{\text {prox }}\right)$ and distal $\left(\mathrm{d}_{\text {distal }}\right)$ probe movement relative to the longitudinal axis of the shank.

This article is protected by copyright. All rights reserved. 
Fig. 3 Left: averaged ( \pm standard deviation $(\mathrm{SD})$ ) fascicle lengths of the medial gastrocnemius $\left(\mathrm{FAS}_{\mathrm{GM}}, \mathrm{N}=18\right)$ and soleus muscles ( $\mathrm{FASC}_{\mathrm{SOL}}, \mathrm{N}=12$ ) time-normalized to the ground contact of the conditioned (HOP; solid line) and control jumps (CTRL; dotted line) of experiment 1; right: $t$ statistics (SPM $\{t\})$ for time-normalized corresponding scalar fields on the right-hand side. Dotted lines represent the threshold of the critical $t$ value $\left(t^{*}\right), p$ values are given if $\operatorname{SPM}\{t\}$ exceeds $t^{*}(\mathrm{p}<0.05$, gray area).

Fig. 4 Left: averaged $( \pm \mathrm{SD})$ length of the passive elastic structures of the medial gastrocnemius ( $\mathrm{L}_{\text {tendon GM}} ; \mathrm{N}=18$ ), and soleus ( $\mathrm{L}_{\text {tendon SOL }} ; \mathrm{N}=12$ ) time-normalized to the ground contact of the conditioned (HOP; solid line) and control jumps (CTRL; dotted line) of experiment 1; right: $t$ statistics $(\operatorname{SPM}\{\mathrm{t}\})$ for time-normalized corresponding scalar fields on the right-hand side. Dotted lines represent the threshold of the critical $t$ value $\left(t^{*}\right), \mathrm{p}$ values are given if $\operatorname{SPM}\{\mathrm{t}\}$ exceeds $t(\mathrm{p}<0.05$, gray area).

Fig. 5 A - left: averaged $( \pm \mathrm{SD})$ force acting on the insertion of the triceps surae muscle $\left(\mathrm{F}_{\text {tendon }}\right)$ time-normalized to the ground contact of the conditioned (HOP; solid line) and control jumps (CTRL; dotted line) of experiment $1(\mathrm{~N}=18)$; A - right: $t$ statistics $(\operatorname{SPM}\{t\})$ for time-normalized corresponding scalar fields on the right-hand side. Dotted lines represent the threshold of the critical $t$ value $\left(t^{*}\right), \mathrm{p}$ values are given if $\operatorname{SPM}\{\mathrm{t}\}$ exceeds $t^{*}(\mathrm{p}<0.05$, gray area). B: mean $( \pm \mathrm{SD})$ mechanical work done by the medial gastrocnemius ( $\mathrm{W}_{\mathrm{MTU} \text { GM }}, \mathrm{N}=18$ ) and soleus ( $\left.\mathrm{W}_{\mathrm{MTU} \mathrm{SOL}}, \mathrm{N}=12\right)$ muscles during the eccentric (ecc) and concentric (conc) phases of the jump. \# indicates a significant difference $(\mathrm{p}<0.05)$ in the Bonferroni corrected post hoc test between HOP and CTRL.

Fig. 6 Left: averaged $( \pm \mathrm{SD})$ Achilles tendon length $\left(\mathrm{L}_{\text {Achilles } \mathrm{GM}}\right)$, averaged $( \pm \mathrm{SD})$ force acting on the insertion of the triceps surae muscle $\left(\mathrm{F}_{\text {tendon }}\right)$, and averaged $( \pm \mathrm{SD})$ external mechanical power of the triceps surae $\mathrm{MTU}\left(\mathrm{P}_{\mathrm{MTU}}\right)$ time-normalized to the ground contact of the conditioned (HOP; solid line) and control jumps (CTRL; dotted line) of

This article is protected by copyright. All rights reserved. 
experiment 1; right: $t$ statistics (SPM $\{\mathrm{t}\})$ for time-normalized corresponding scalar fields. Dotted lines represent the threshold of the critical $t$ value $\left(t^{*}\right), \mathrm{p}$ values are given if $\operatorname{SPM}\{\mathrm{t}\}$ exceeds $t^{*}(\mathrm{p}<0.05$, gray area).

Fig. 7: Mean $( \pm \mathrm{SD})$ mechanical work derived from the Achilles tendon strain energy of the

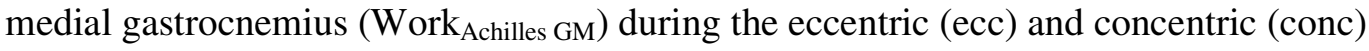
phases of the jump. \# indicates a significant difference $(\mathrm{p}<0.05)$ in the Bonferroni corrected post hoc test between HOP and CTRL.

This article is protected by copyright. All rights reserved. 


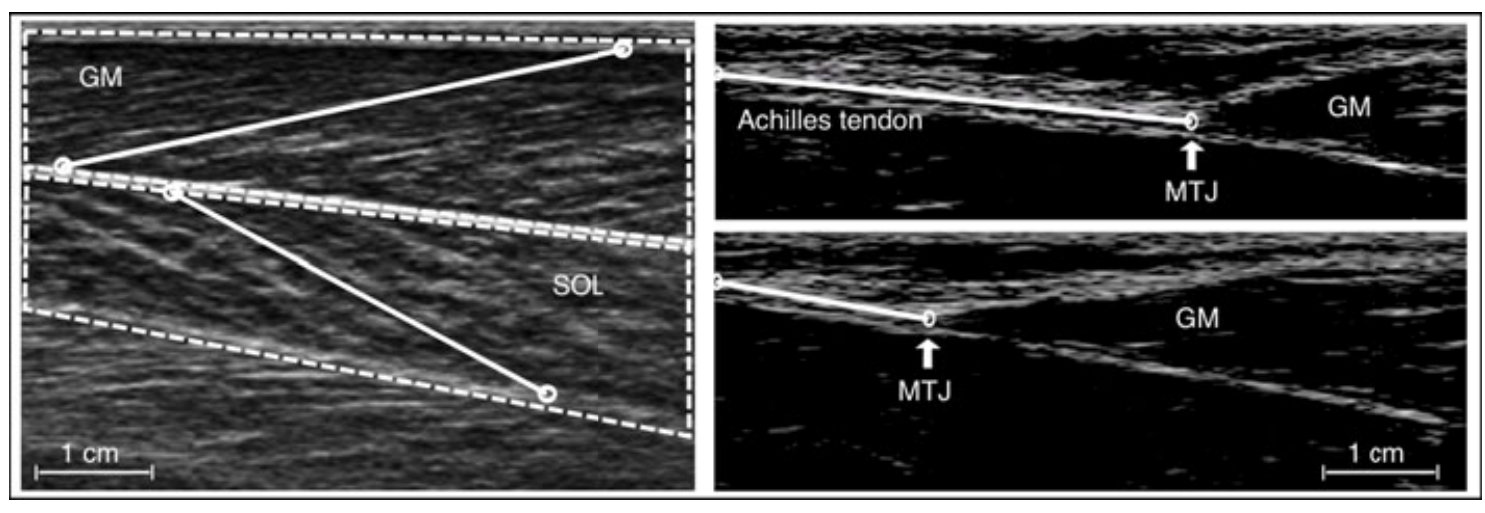

Fig. 1: Left: Fascicle length tracking of the ultrasound images of the medial gastrocnemius (GM, upper dotted box) and soleus (SOL, lower dotted box) between the superficial and deeper aponeuroses for each muscle during drop jumps. Note: the contrast has been reduced in this picture for better perceptibility of the tracked fascicles (solid lines). Right: Tracking of the muscle tendon junction (MTJ) between the GM and the proximal Achilles tendon (solid lines). The upper and lower pictures denote the amplitude of MTJ movement during a drop jump. Note: total Achilles tendon length was calculated by adding the actual tracked length to the distance between the distal end of the ultrasound probe and the Achilles tendon insertion on the calcaneus.

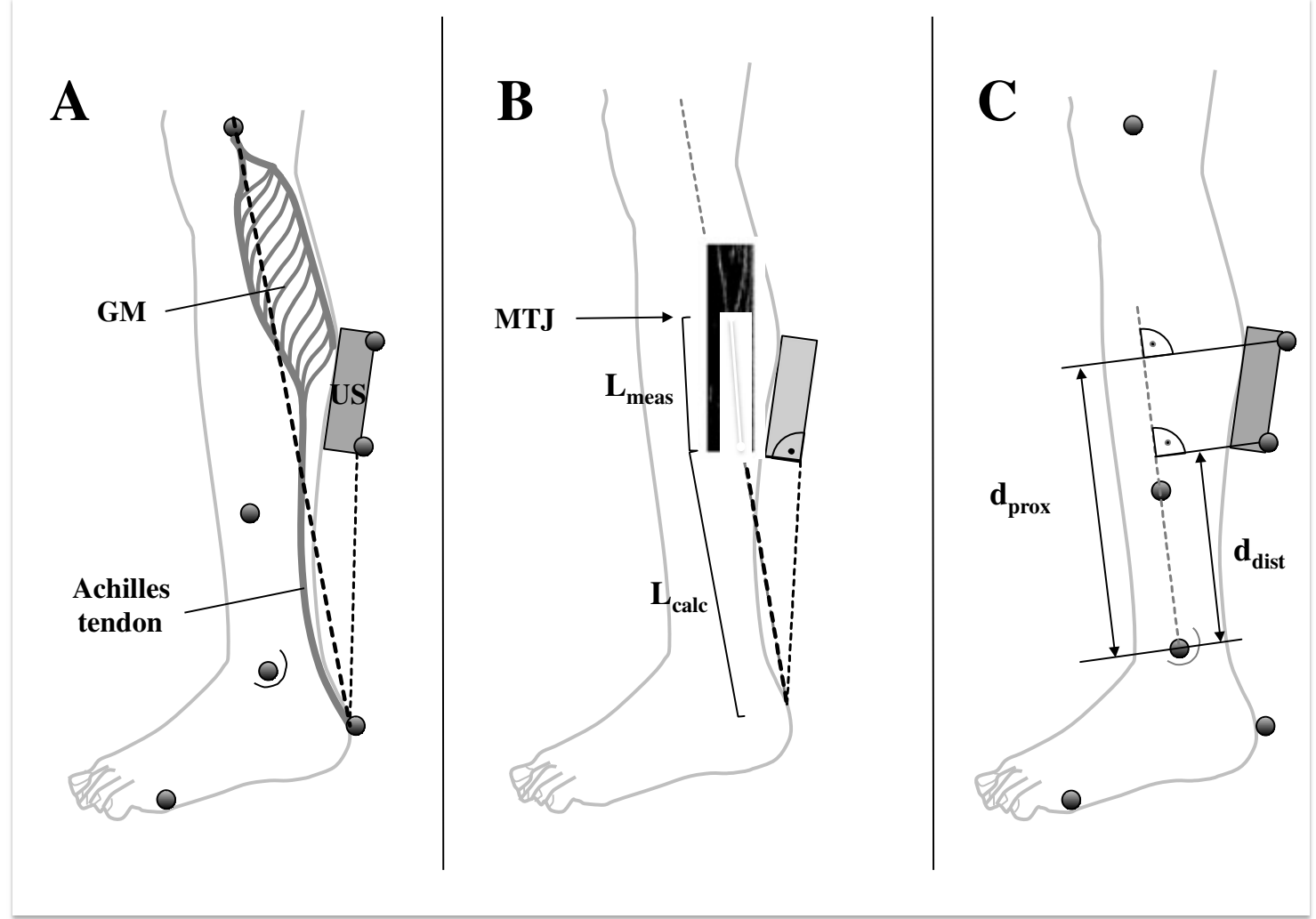

Fig. 2 A: The distance between the ultrasound probe and Achilles tendon was estimated with the help of motion capture markers attached to the probe and to the calcaneus. This distance was projected to the instantaneous working axis of the medial gastrocnemius (GM) between its origin and insertion. B: This projection revealed the calculated part of the Achilles tendon length $\left(\mathrm{L}_{\mathrm{calc}}\right)$, which was added to 
the measured length of the tendon $\left(\mathrm{L}_{\text {meas }}\right)$ via tracking of the muscle tendon junction (MTJ). $\mathrm{C}: \mathrm{L}_{\text {meas }}$ was corrected for the proximal $\left(\mathrm{d}_{\text {prox }}\right)$ and distal $\left(\mathrm{d}_{\mathrm{distal}}\right)$ probe movement relative to the longitudinal axis of the shank.
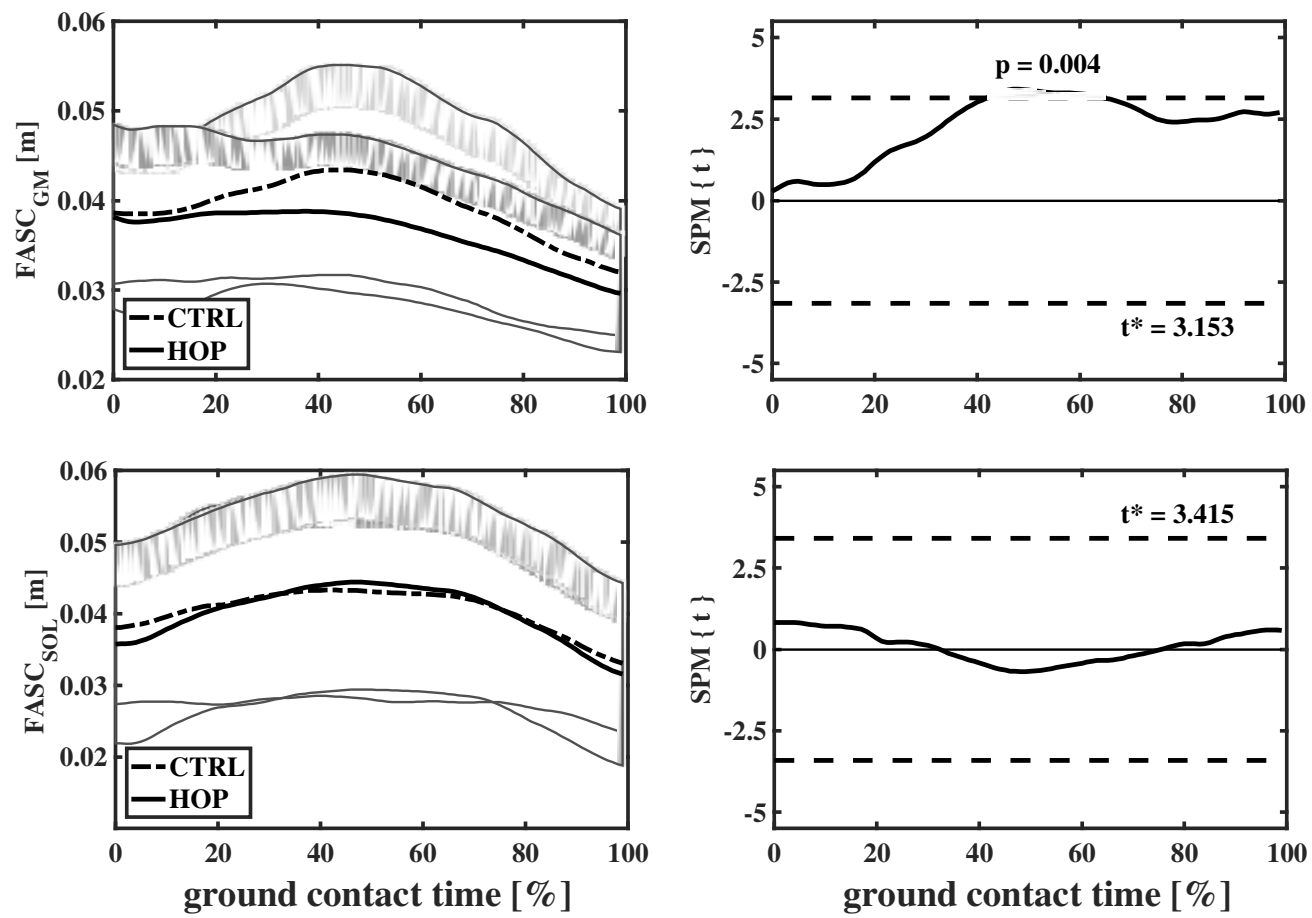

Fig. 3: Left: averaged fascicle lengths of the medial gastrocnemius $\left(\mathrm{FAS}_{\mathrm{GM}}, \mathrm{N}=18\right)$ and soleus muscles $\left(\mathrm{FASC}_{\mathrm{SOL}}, \mathrm{N}=12\right.$ ) time-normalized to the ground contact of the conditioned (HOP; solid line) and control jumps (CTRL; dotted line) of experiment 1; right: $t$ statistics (SPM $\{t\}$ ) for timenormalized corresponding scalar fields on the right-hand side. Dotted lines represent the threshold of the critical $t$ value $\left(t^{*}\right), p$ values are given if $\operatorname{SPM}\{t\}$ exceeds $t^{*}(\mathrm{p}<0.05$, gray area).

This article is protected by copyright. All rights reserved. 

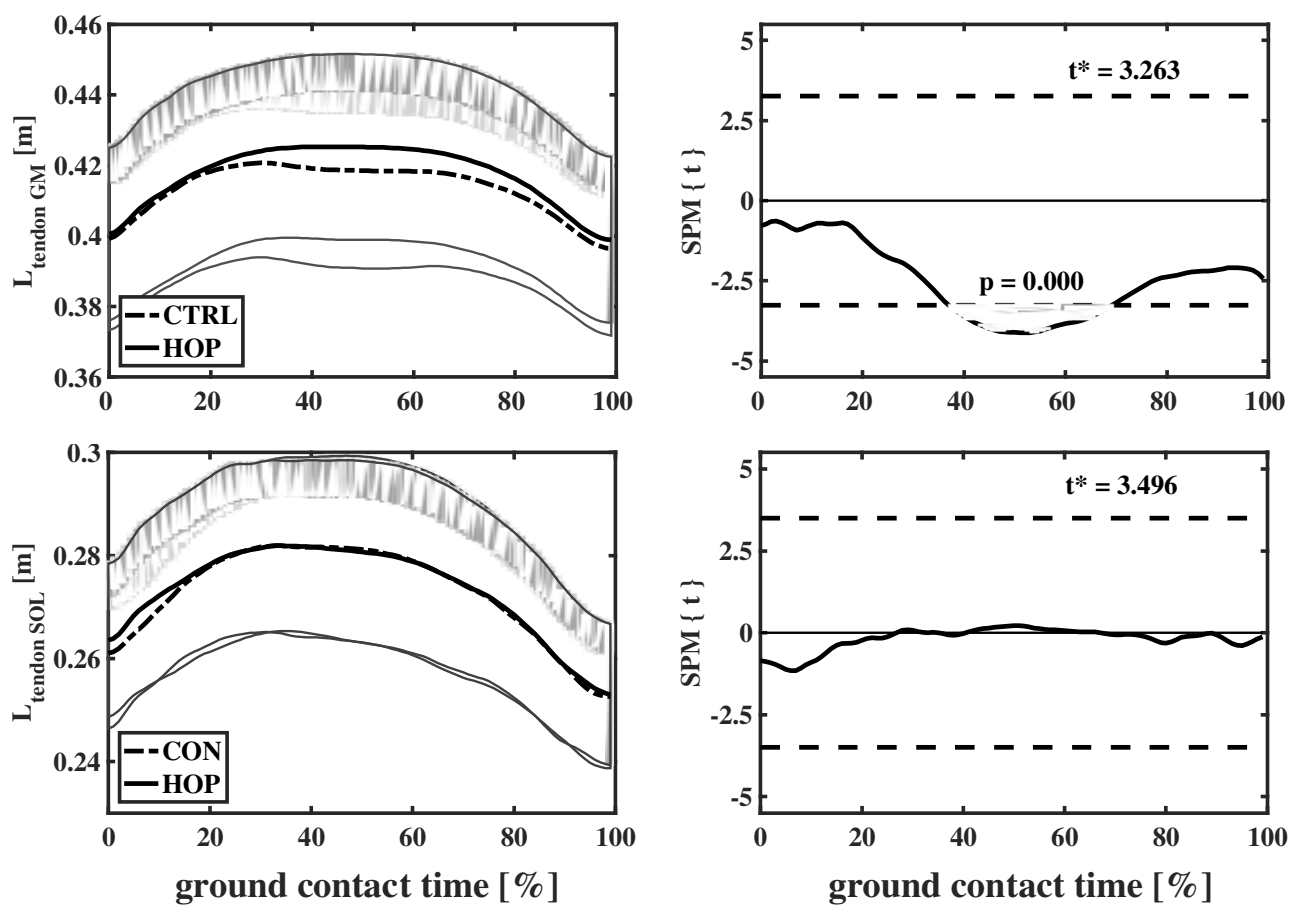

Fig. 4: Left: averaged length of the passive elastic structures of the medial gastrocnemius $\left(\mathrm{L}_{\text {tendon } \mathrm{GM}}\right.$; $\mathrm{N}=18$ ), and soleus ( $\mathrm{L}_{\text {tendon }} \mathrm{SOL} ; \mathrm{N}=12$ ) time-normalized to the ground contact of the conditioned (HOP; solid line) and control jumps (CTRL; dotted line) of experiment 1; right: $t$ statistics (SPM $\{t\}$ ) for time-normalized corresponding scalar fields on the right-hand side. Dotted lines represent the threshold of the critical $t$ value $(t *), \mathrm{p}$ values are given if $\mathrm{SPM}\{\mathrm{t}\}$ exceeds $t(\mathrm{p}<0.05$, gray area).

This article is protected by copyright. All rights reserved. 

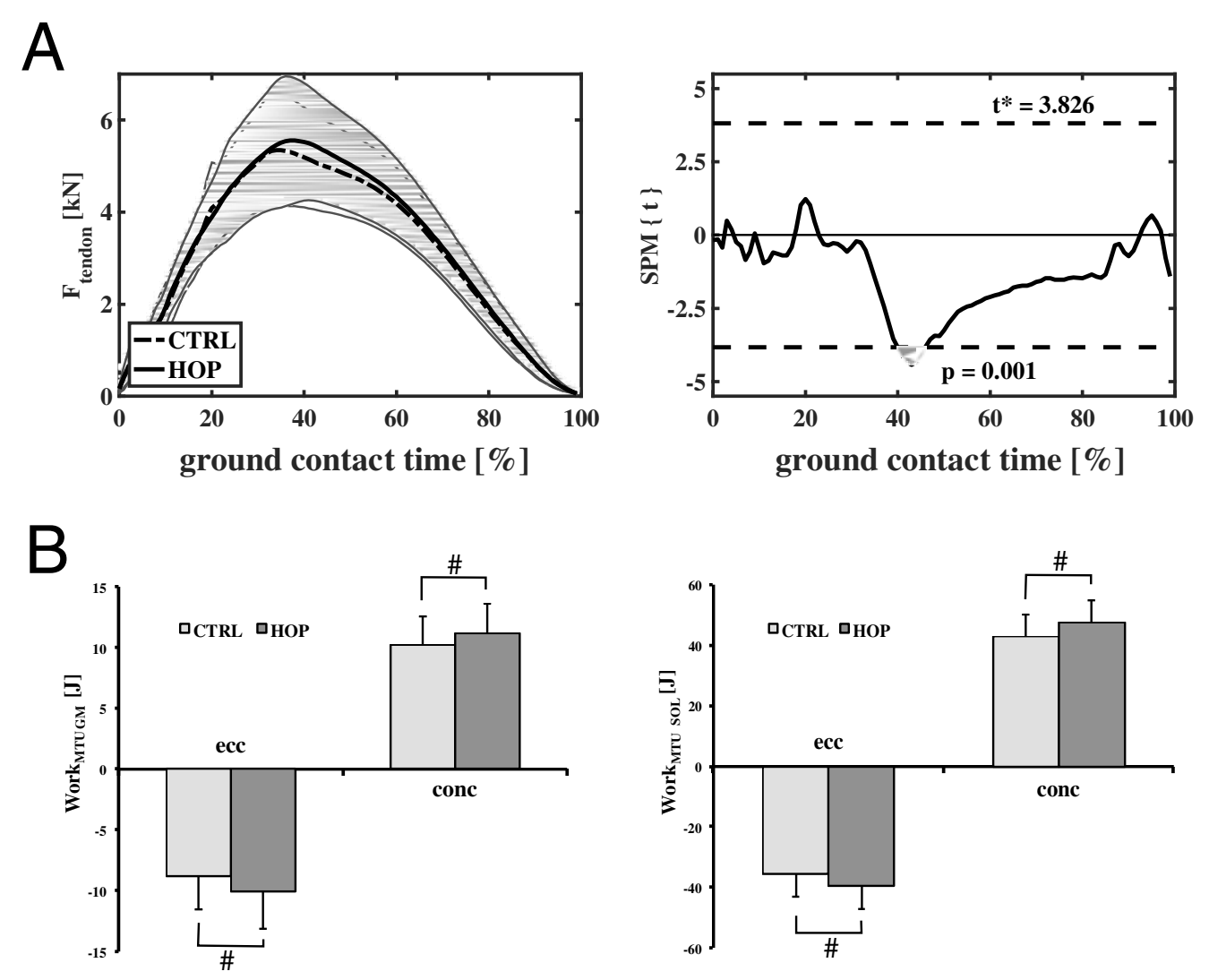

Fig. 5: A - left: averaged force acting on the insertion of the triceps surae muscle $\left(\mathrm{F}_{\text {tendon }}\right)$ timenormalized to the ground contact of the conditioned (HOP; solid line) and control jumps (CTRL; dotted line) of experiment $1(\mathrm{~N}=18)$; A - right: $t$ statistics (SPM $\{t\})$ for time-normalized corresponding scalar fields on the right-hand side. Dotted lines represent the threshold of the critical $t$ value $\left(t^{*}\right), \mathrm{p}$ values are given if $\mathrm{SPM}\{\mathrm{t}\}$ exceeds $t^{*}(\mathrm{p}<0.05$, gray area). B: mechanical work done by the medial gastrocnemius ( $\mathrm{W}_{\mathrm{MTU}} \mathrm{GM}, \mathrm{N}=18$ ) and soleus ( $\left.\mathrm{W}_{\mathrm{MTU} \mathrm{SOL}}, \mathrm{N}=12\right)$ muscles during the eccentric (ecc) and concentric (conc) phases of the jump. \# indicates a significant difference $(\mathrm{p}<0.05)$ in the Bonferroni corrected post hoc test between HOP and CTRL.

This article is protected by copyright. All rights reserved. 

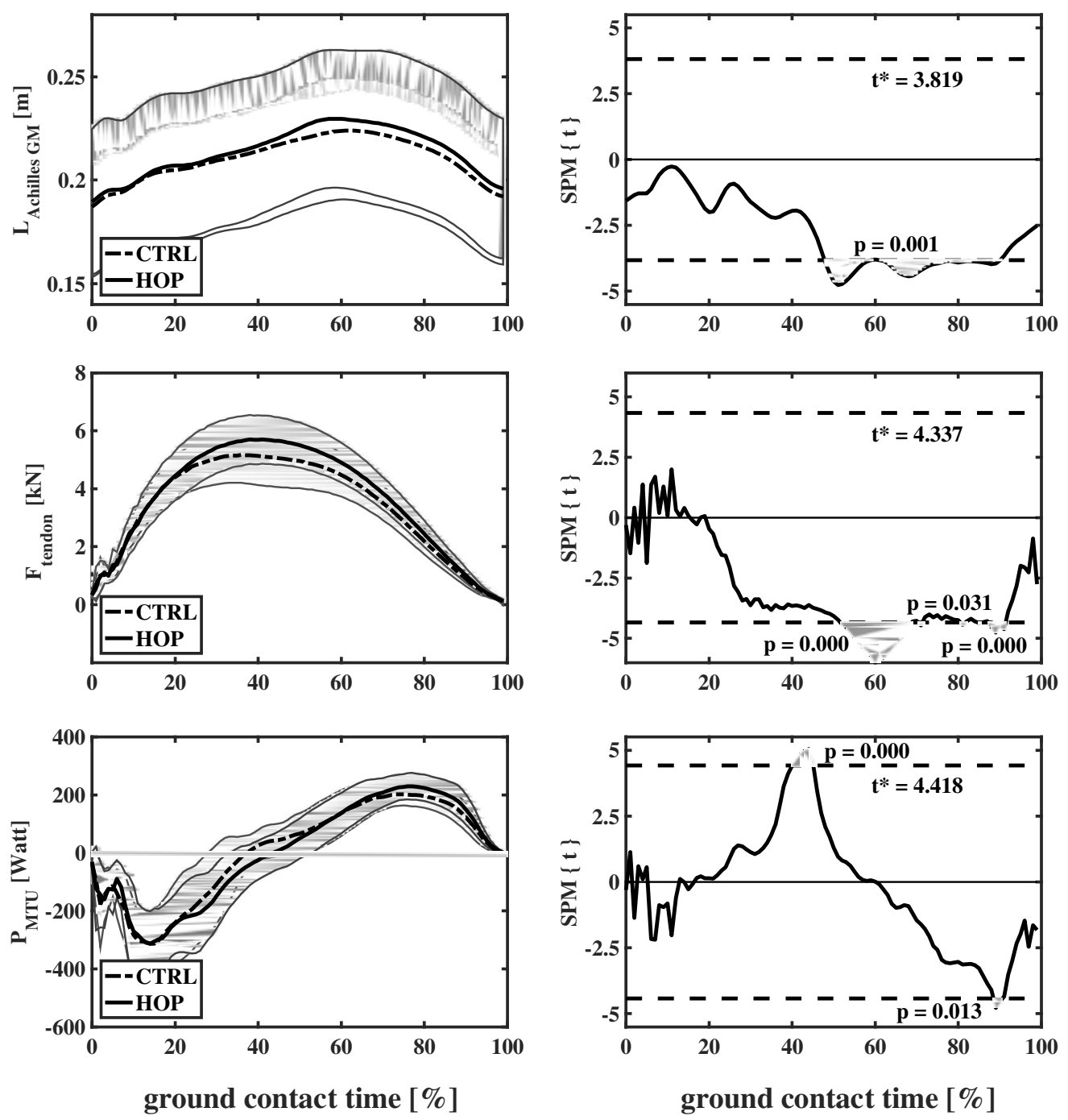

Fig. 6: Left: averaged Achilles tendon length $\left(\mathrm{L}_{\text {Achilles } \mathrm{GM}}\right)$, force acting on the insertion of the triceps surae muscle $\left(\mathrm{F}_{\text {tendon }}\right)$, and external mechanical power of the triceps surae MTU ( $\left.\mathrm{P}_{\mathrm{MTU}}\right)$ timenormalized to the ground contact of the conditioned (HOP; solid line) and control jumps (CTRL; dotted line) of experiment 1; right: $t$ statistics (SPM $\{\mathrm{t}\})$ for time-normalized corresponding scalar fields. Dotted lines represent the threshold of the critical $t$ value $\left(t^{*}\right), \mathrm{p}$ values are given if SPM $\{\mathrm{t}\}$ exceeds $t^{*}(\mathrm{p}<0.05$, gray area).

This article is protected by copyright. All rights reserved. 


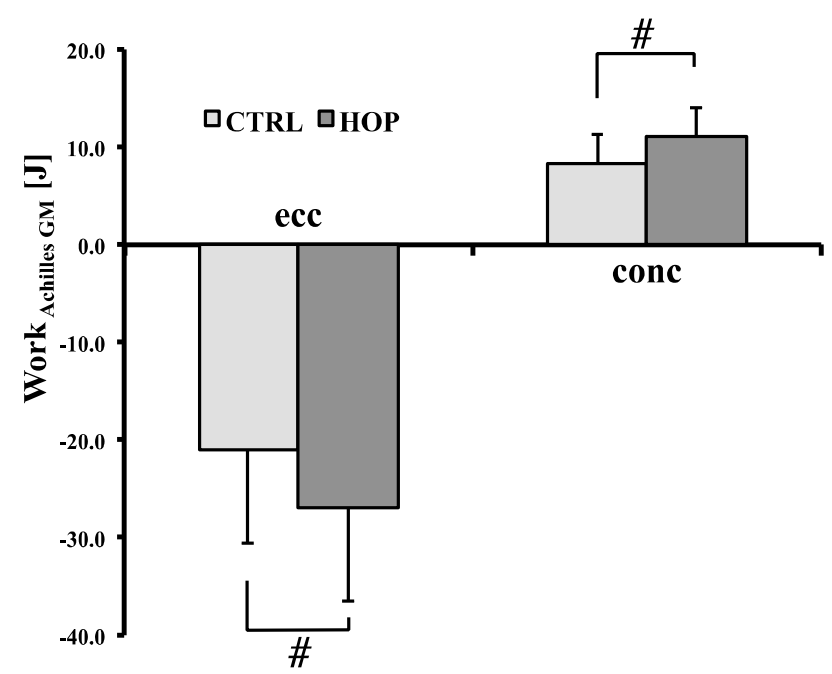

Fig. 7: Mechanical work derived from the Achilles tendon strain energy of the medial gastrocnemius

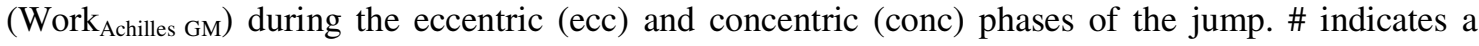
significant difference $(\mathrm{p}<0.05)$ in the Bonferroni corrected post hoc test between HOP and CTRL.

This article is protected by copyright. All rights reserved. 